\title{
Application of Taguchi's Methods for Optimizing Organic Rankine Cycle for Recovering Low Grade Industrial Waste Heat
}

\author{
Umesh Kumar, Munawar N. Karimi \\ Jamia millia Islamia,New Delhi, India, 110025
}

\begin{abstract}
This research attempts the optimization of Organic Rankine Cycle (ORC) by Taguchi's robust design methods for generating electricity from industrial waste heat. Experimental results and data from previous researches is used to assign the relative importance of parameters. Parameters selected are waste stream temperature, waste stream flow rate, type of refrigerant and turbine speed. Optimum combination of these control parameters is obtained by using Taguchi's techniques. Individual effect of parameters on the performance of Organic Rankine Cycle is calculated. Interaction effect of parameters on the performance of ORC is also calculated. A percent effect of individual parameters on performance is estimated by using ANOVA. Using this procedure, an optimal cycle can be obtained for maximum efficiency within available sources.
\end{abstract}

Keywords: Electricity generation; Waste heat recovery; Optimization; Taguchi's method; Organic Rankine cycle; ANOVA

\section{Introduction}

Waste heat is a byproduct of energy conversion processes. It is a thermodynamic obligation which cannot be avoided. Waste heat is directly released into the environment, which has direct and adverse effects on economics and environment. As the fossil fuel resources are depleting very fast, it becomes mandatory for industries to capture this waste heat and to use it logically. Recovery and use of waste heat may reduce energy costs and improves the profitability of any operation[1].Though high temperature waste heat is often reused to perform work or for heating purposes but the use and recovery of low temperature waste heat is still not in regular practice. In industries throughout the world low temperature waste heat amounts significant losses.

Waste heat can be recovered in many ways such as by using heat exchanger, heat pump and energy recovery wheels. All of these methods require a utilization point where the recovered heat can be used. In the absence of utilization point any effort of heat recovery has no meaning. So, it puts a constraint on the recovery of waste heat as it may not always possible to find a suitable utilization point in the nearby vicinity of waste heat source. Power generation from recovered heat provides a better option as electricity is always in demand everywhere. The conversion of heat recovered at lower temperature into

* Corresponding author.

E-mail: umesh_1977us@yahoo.com

(c) 2014 International Association for Sharing Knowledge and Sustainability

DOI: $10.5383 /$ ijtee.08.02.005 electricity cannot be achieved by normal steam cycles. Organic Fluids and refrigerants are used for this purpose and the cycle is known as Organic Rankine Cycle. The main difference between organic fluids and water is the lower evaporation energy of organic fluids. So, less heat is required to evaporate the organic fluid. It ensures that low temperature waste heat of $80^{\circ} \mathrm{C}$ to $100^{\circ} \mathrm{C}$ can also be used as a heat source to the ORC. Efficiencies of ORC as compared to Rankine steam cycle are very low.

Various researchers have attempted to optimize the ORC operations. Agustin et al. [2] tried to optimize the low temperature ORC run by solar heat. Florian Heberie et al. [3] performed an exergy based analysis for selecting the optimized fluid for a geothermal ORC. Sylvain Quoilin et al. [4] presented a dynamic model to describe the behavior of ORC in a variable flow rate and temperature heat source and formulated a control strategy to optimize the cycle. The cycle was tested with five different working fluids and a comparison was presented. Bertrand Fankam Tchanche et al. [5] compared 20 different refrigerants for relevant parameters and estimated their suitability for ORC. P J Mago [6] presented a Second-law analysis of some organic working fluids for the use of ORC, which were R134a, R113, R245ca, R245fa, R123, Iso-butane, and propane, with boiling points between 24.3 and $48.8^{\circ} \mathrm{C}$. 


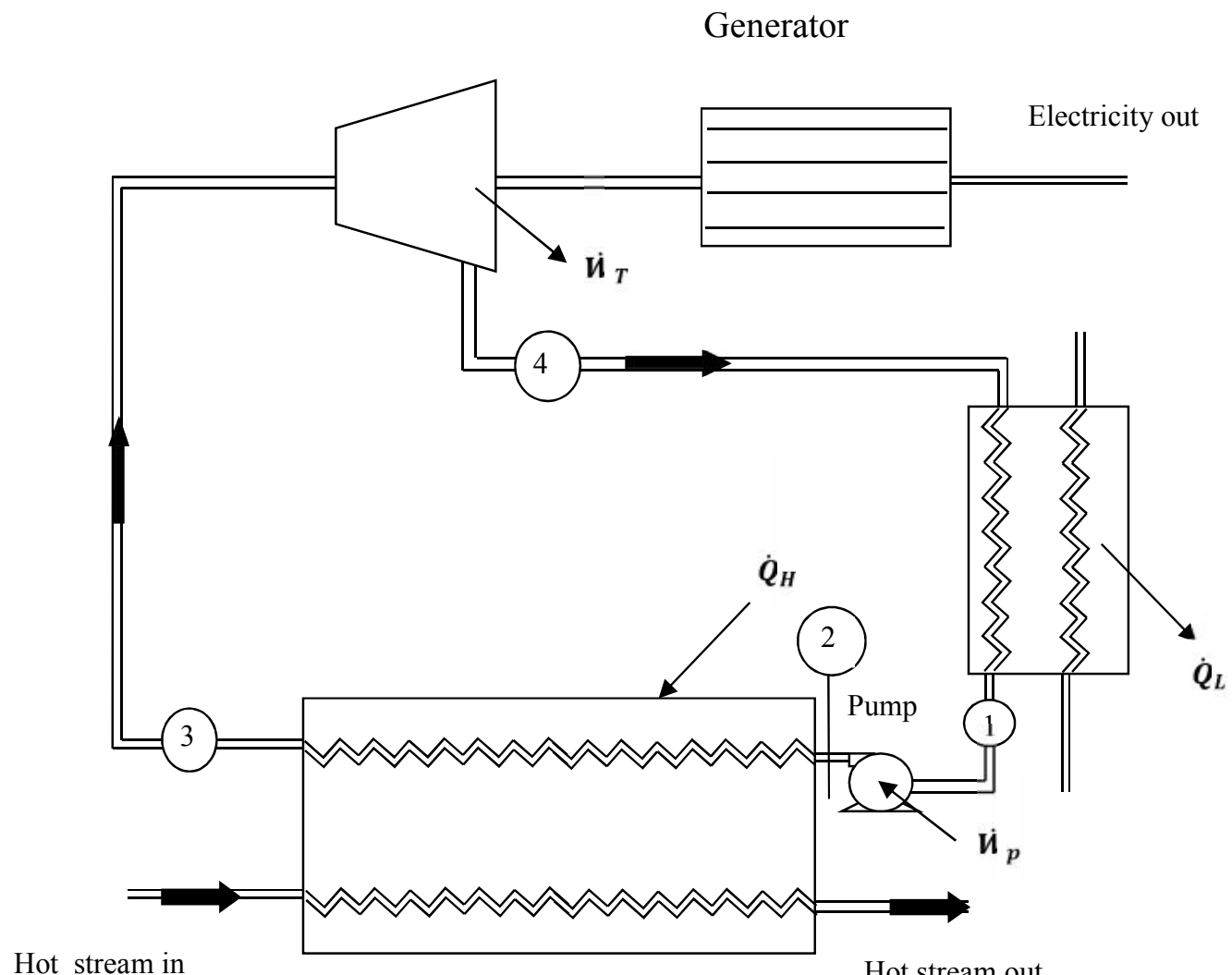

Hot stream in

Hot stream out

Fig. 1: Schematic of Organic Rankine Cycle utilizing waste heat stream

\section{Performance Parameters}

Performance of Organic Rankine Cycle is governed by a large number of operating parameters. Individual as well as combined effect of these parameters is important to optimize the overall working of ORC. Researchers have tried to optimize the ORC, most of these researches have taken one parameter at a time but combined effect of all the parameters is essential for system optimization. Tianyou Wang et al. [7] reviewed the researches on heat recovery with Rankine cycle and investigated various different system configurations to arrive at highest possible system efficiency. Adrienne B. Little and Sriniwas Garimella [8] attempted a comparative assessment of alternative cycles for waste heat recovery and found that Organic Rankine cycle performed better than other alternatives.

This paper evaluates the combined effect of important parameters and their variation on the cycle outcome and finally gives a thorough guideline towards selecting an optimized cycle. A detailed literature survey was attempted in this regard to identify the different optimization techniques used by different researchers. Commonly used methods include Genetic Algorithms, Dynamic Modeling and Parametric Optimization method. All of these techniques consider one parameter at a time and help in optimizing that parameter for obtaining the maximum output, these methods do not consider the simultaneous effect of other parameters on the cycle outcomes. An attempt is made to optimize the ORC by optimizing the combined effect of control parameters. Effects of individual control parameters are also evaluated by using Taguchi's methods. ANOVA (analysis of variance) technique is used to evaluate the importance of factors and their optimized combination.

\section{Methodology}

Taguchi's robust design approach is used to analyse and optimize the parameters of Organic Rankine cycle run with low temperature waste heat energy.

\subsection{Taguchi's Method}

Taguchi's method is a powerful tool for the design of high quality systems. It provides simple, efficient and systematic approach to optimise designs for performance quality [9]. The methodology is valuable when the design parameters are qualitative and discrete. It can optimise the performance characteristics through the setting of the design parameters and reduce the sensitivity of the system performance to the sources of variation. In Taguchi's method the engineering optimization of a process/product is carried out in three steps approach that is system design, parameter design and tolerance design[10]. In the process optimization, system 
design is fixed from the thermal design input. Tolerance design is related to manufacturing, which is not in scope of thermal process systems. Therefore, Taguchi approach for parametric design is used for the present work. Taguchi's parameter design optimizes the performance characteristics by the settings of design parameters. In recent years Taguchi's method has been applied to a number of applications in a wide range of industries but the application of Taguchi's method for energy recovery is limited till date[10]. In Present work taguchi's methods are introduced to engineering processes to optimize waste heat recovery by using ORC.

\section{System Description}

The ORC uses an organic fluid as a working fluid. The cycle consists of five components: an evaporator, a pump, an expander, a condenser, and a working fluid. The evaporator absorbs heat into the cycle and condenser releases it from the cycle. The cycle is started when the pump pushes the working fluid to the evaporator.

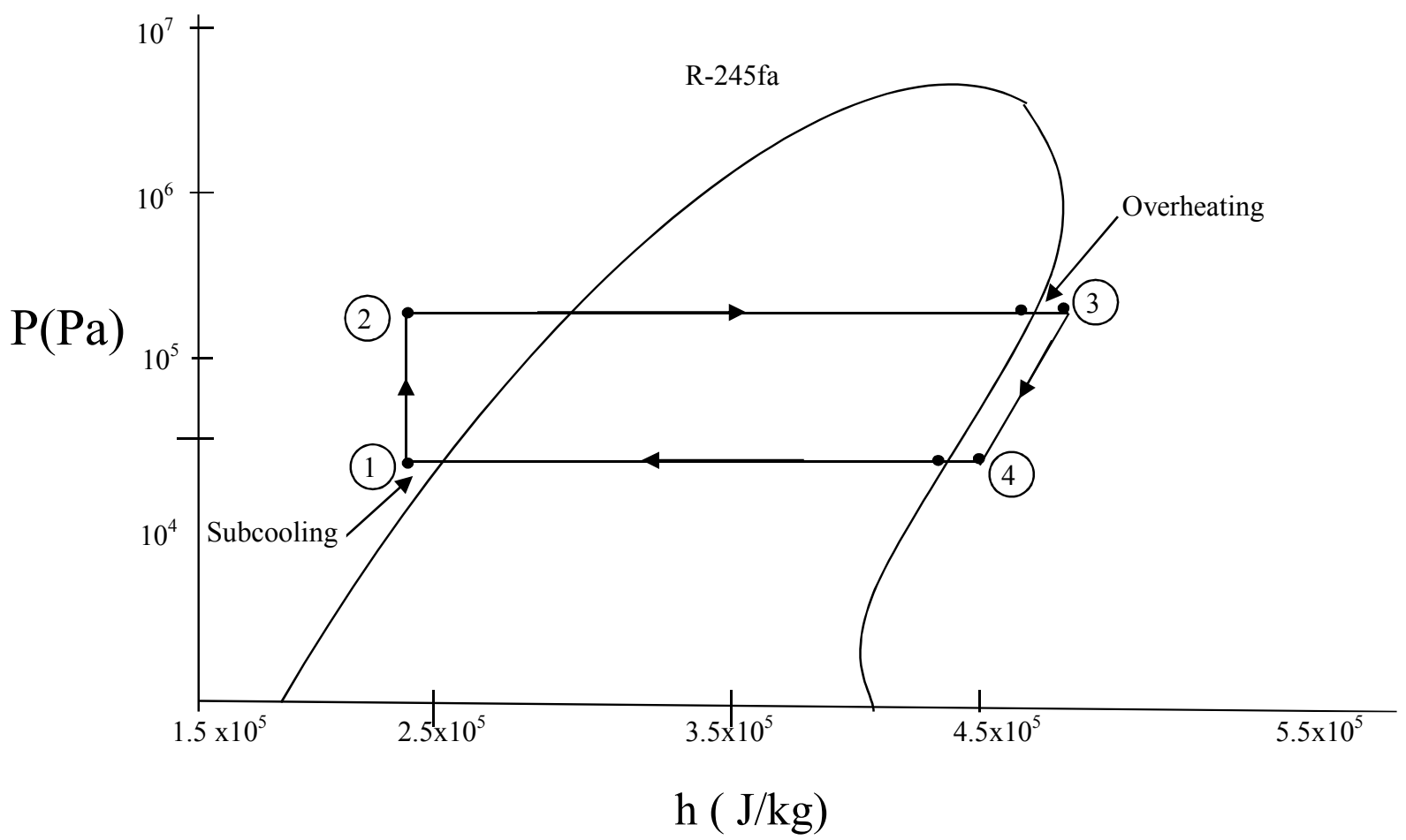

Fig. 2. Chart depicting Organic Rankine cycle on p-h diagram for R-245fa

In the evaporator, the fluid at high temperature source (waste heat here) heats the working fluid to a saturated or superheated vapor state. Then, the vapour expands in the expander and rotates it to produce power.

After the vapor leaves the turbine, the water at the cold source cools and condenses the working fluid into the liquid state in the condenser. Then, the pump re-circulates the fluid. Fig. 2 shows the pressure-enthalpy $(\mathrm{p}-\mathrm{h})$ diagram corresponding to Fig. 1 The efficiency of Organic Rankine cycle can be used in different conditions and for different combination of operating parameters. P.J Mago et al.[6] describe the relations used for calculating first law and second law efficiencies along with the processes.

\section{Process 1-2 (Pump)}

Pump drives the Organic Rankine Cycle. Pump power is expressed as

$$
W_{p}=\frac{W_{p, \text { ideal }}}{\eta_{p}}=\frac{\dot{m}\left(h_{1}-h_{2 s}\right)}{\eta_{p}}
$$

$\mathrm{W}_{\text {p.ideal }}$ is ideal power of pump. is working fluid mass flow rate. $\eta_{p}$ is isentropic efficiency of the pump. $h_{1}$ and $h_{2 s}$ are the enthalpies of working fluid at inlet and outlet of pump respectively. The irreversibility rate for uniform flow condition is

$\dot{I}=T_{o} \frac{d s}{d t}$

Irreversibility rate for the pump is

$\dot{I}_{p}=T_{o} \dot{m}\left(s_{2}-s_{1}\right)$

$s_{1}$ and $s_{2}$ represent the specific entropies of working fluid at inlet and exit of the pump. 


\section{Process 2-3 ( evaporator)}

2-3 process is isobaric heat transfer. Working fluid is heated in evaporator. It is situated between pump outlet and turbine inlet. Input to the turbine can be saturated or superheated vapour. Heat transfer rate is given by

$\dot{Q}_{e}=\dot{m}\left(h_{3}-h_{2}\right)$

$h_{3}$ and $h_{2}$ are the enthalpies of the working fluid at the exit and inlet of the evaporator respectively. Evaporator irreversibility rate is given as

$\dot{I}_{e}=T_{o} \dot{m}\left[\left(s_{3}-s_{2}\right)-\frac{h_{3}-h_{2}}{T_{H}}\right]$

$s_{3}$ and $s_{2}$ are the specific entropies of working fluid at inlet and exit of evaporator respectively. $\mathrm{T}_{\mathrm{H}}$ represents the temperature of hot stream.

\section{Process 3-4 ( Turbine)}

Mechanical power is generated in turbine when the working fluid passé through it. The turbine power is

$\dot{W}_{t}=\dot{W}_{t, \text { ldeal } \eta_{t}}=\dot{\dot{m}}\left(h_{3}-h_{4 s}\right) \eta_{t}$

$\dot{W}_{t, \text { ideal } \eta_{t}}$ is the ideal power of the turbine, $\eta_{\mathrm{t}}$ is turbine isentropic efficiency, $h_{3}$ and $h_{4 s}$ are the enthalpies of working fluid at inlet and outlet of the turbine. Turbine irreversibility rte is given by

$\dot{I}_{t}=T_{o} \dot{m}\left(s_{4}-s_{3}\right)$

$\mathrm{s}_{3}$ and $\mathrm{s}_{4}$ are the specific entropies of working fluid at the inlet and the outlet of the turbine respectively.

\section{Process 4-1 (Condenser)}

Constant pressure phase change takes place in condenser and working fluid is converted into saturated liquid. Condenser heat rate is given as

$\dot{Q}_{c}=\dot{m}\left(h_{1}-h_{4}\right)$

Condenser irreversibility is given by

$\dot{I}_{c}=T_{o} \dot{m}\left[\left(s_{1}-s_{4}\right)-\frac{h_{1}-h_{4}}{T_{L}}\right]$

$\mathrm{s}_{1}$ and $\mathrm{s}_{4}$ represent specific entropies of working fluid at inlet and outlet of the condenser respectively. $T_{L}$ is the temperature of cold reservoir.

\section{Efficiency of Organic Rankine Cycle}

Thermal efficiency is the ratio of net power of cycle to evaporator heat rate.

$\eta_{t h}=\frac{\dot{W}_{t}+\dot{W}_{p}}{\dot{Q}_{e}}$

In waste heat recovery applications the objective is to improve global efficiency rather than improving thermal efficiency. Global efficiency is given as

$\eta_{\text {global }}=\frac{\dot{W}_{t}+\dot{W}_{p}}{Q_{\text {avaltable }}}=\varepsilon_{\text {rec }} \cdot \eta_{\text {th }}$

$\varepsilon_{\text {rec }}$ is the efficiency of heat recovering in evaporator and is given as $\varepsilon_{\text {rec }}=\frac{\dot{Q}_{\text {recovered }}}{\dot{Q}_{\text {avaitable }}}$

The global efficiency is to be maximized here. The operating conditions which maximize the global efficiency are different from those who maximize the efficiency of ORC [4]. Global efficiency is taken as the Performance evaluation parameter for ORC. Taguchi's Robust Design method is applied for an ORC having five variable parameters. Two levels of each parameter are considered for optimizing the cycle.

\subsection{Cycle Parameters:}

\section{A. Turbine Speed:}

Selection and design of turbine is based on maximum efficiency, small footprint and minimum speed. The design point rotational speed is set by generator speed and mechanical coupling between the two. The expander wheel is designed at the optimum specific speed $\mathrm{N}_{\mathrm{s}}$.

$$
N_{s}=\frac{76 . N}{1000} \cdot \sqrt{\frac{Q_{s}}{\sqrt{\Delta H_{i s}{ }^{3}}}}
$$

Figure 4 from balje (1980) describes the range of specific speeds for different types of turbines.

Two levels of turbine speeds are considered for the analysis.

\section{B. Waste stream temperature:}

Waste heat is categorized in three categories viz. high, medium and low grade waste heats depending upon the temperatures. High grade waste heat has temperature ranges from $1350^{\circ} \mathrm{C}$ and above. Medium grade waste heat temperature varies from $230^{\circ} \mathrm{C}$ to $1350^{\circ} \mathrm{C}$ and below $230^{\circ} \mathrm{C}$ is termed as low grade waste heat. Recovery of high grade and medium grade waste heat is a routine practice in industries. The present work considers the low grade waste heat recovery which is generally ignored in most of the industries of developing countries due to marginal benefits and large financial implications.

\section{Refrigerant/ working fluid:}

The selection of working fluid plays a significant role in the Organic Rankine Cycle [11]. The Organic working fluid must be carefully selected on the basis od safety and technical feasibility. There is a wide selection of working fluids that could be used in ORC [3]. Generally, a good working fluid should exhibit low toxicity, good material compatibility and fluid stability limits [12]. A thermodynamic property of a working fluid affects the economics of the cycle. A bad choice can lead to expensive and inefficient operation. Researches are available for assigning the suitability of working fluids. B.F. Tchanche et al.[13] presented the criteria for proper selection of working fluid for ORC applications. Bertrand et al. [5] evaluated the characteristics of twenty potential working fluids and compared them for a $2 \mathrm{~kW}$ micro power system. Only one criterion was chosen for the selection that was critical temperature and a subcritical cycle was considered. Lee et al. [14] found that the system efficiency and the properties of working fluid are correlated. Parameters chosen here for optimization are taken from various researches done in the selection of working fluids. Some of the characteristics of the Organic Fluids which affect the overall performance in terms of fluid selection are Irreversibility, Critical temperature, Cycle Pressure and Environmental aspects. These four characteristics are the attributes for assigning the suitability of a working fluid. 
Dereje S. Ayou et. al. [25] evaluated the performance of different cycle for combined cooling and power generation using different refrigerants mixtures.

\section{Mass flow rate of waste stream:}

Net heat content of a stream depends upon the mass entering per unit time in the system. Waste Heat recovery largely depends upon the amount of heat content in a waste stream. It varies directly with the type of industry. Thermal processing houses such as textile processing and paper mills use large quantities of hot water and a good amount of hot water streams are generally discharged into environment without any heat recovery. Higher mass flow rates result in higher recovering efficiencies and better economies. It also results in small heat exchanger sizes.

\section{E. Condensate Temperature:}

Lower Condensate temperatures helps to achieve higher efficiencies. Two levels of condensate temperatures are taken for the present work which is near to the temperatures which persist in normal weather conditions.

Along with the above parameters the interdependencies of the few factors were also considered

\section{AxC. Interaction effect off refrigerant with expander rotation speed:}

Design of turbine vary as compared to normal water cycle due to change in pressure ratio, flow rate and refrigerant properties. The fluid properties of the refrigerant are different from those of air, exhaust gas and steam. A change in refrigerant may affect the turbine speed for getting the same output. $\mathrm{A} \times \mathrm{C}$ represents how the parameter $\mathrm{A}$ is related to parameter C. Figure 7 represents interaction effect of $\mathrm{A}$ and C. Two parallel lines represents no iteration at all and the variables may be assumed to be independent two intersecting lines show the dependencies between these two and extent of which is directly proportional to the angle between two lines.

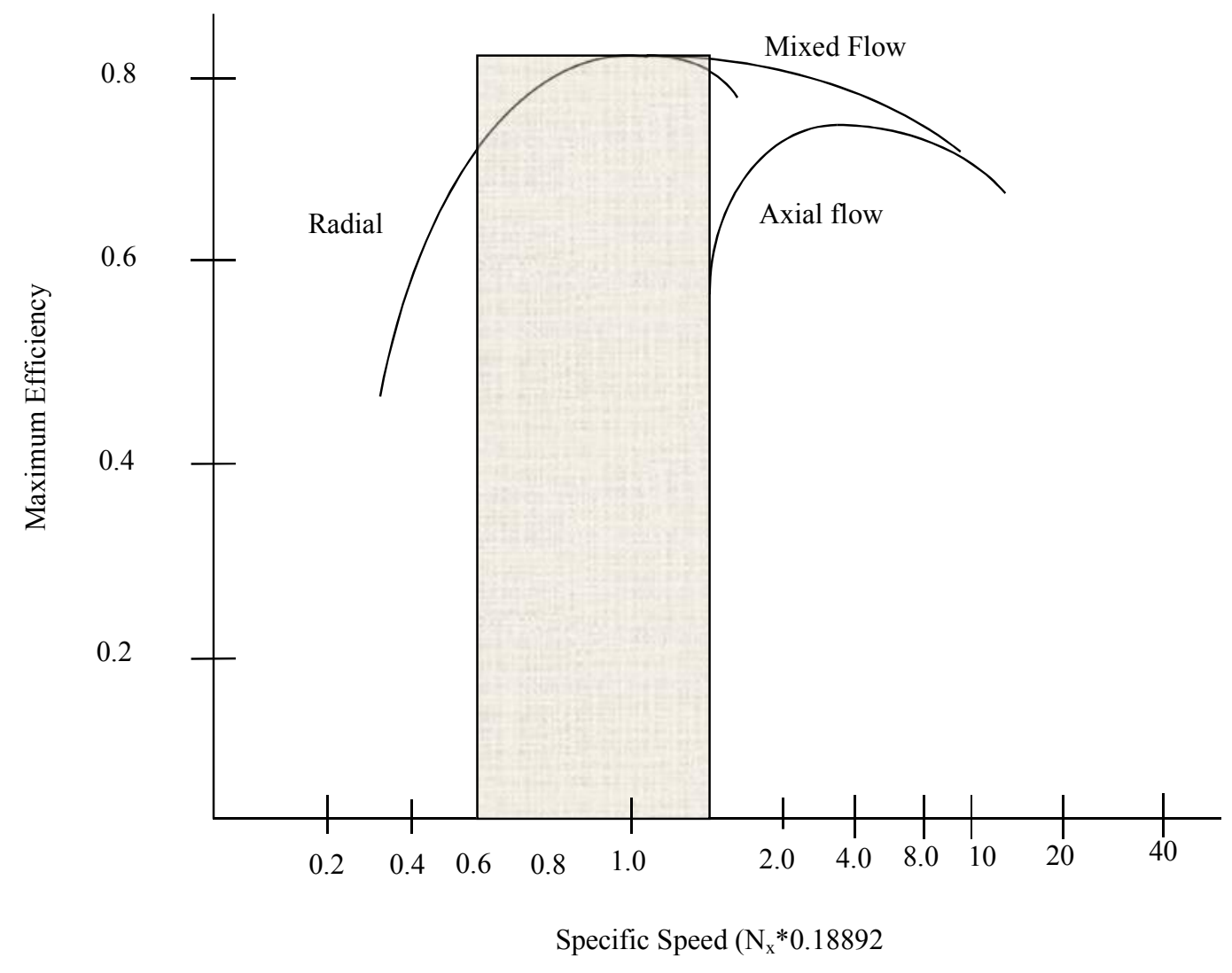

Fig.3. Specific speeds Vs efficiency of various turbine types ( Source: Balje 2008) 
Table 1: Cycle Parameters used in this investigation

\begin{tabular}{|c|l|l|l|}
\hline FACTORS & \multicolumn{1}{|c|}{ DESCRIPTION } & LEVEL 1 & LEVEL 2 \\
\hline A & TURBINE SPEED (RPM $)$ & 3500 & 4500 \\
\hline B & WASTE STREAM TEMPERATURE $\left({ }^{\circ} \mathrm{C}\right)$ & 85 & 95 \\
\hline C & REFRIGERANT & FE7000 & R245FA \\
\hline D & MASS FLOW RATE OF WASTE STREAM & 90 & 60 \\
\hline E & CONDENSATE TEMPERATURE $\left({ }^{\circ} \mathrm{C}\right)$ & & \\
\hline
\end{tabular}

$B \times C$. Interaction effect of waste heat temperature with refrigerant:

Choice of refrigerant largely depends upon the temperature of waste stream. Figure 8 represents the interaction of B and C.

\section{Taguchi's Method}

Dr. Taguchi developed a methodology to ensure the optimum combination of operating parameters using Orthogonal Arrays. Design of experiments (DOE) approach is introduced to optimization of control parameters of Organic Rankine cycle in order to obtain the best efficiencies. Taguchi's method ensures it by minimizing the variance and S/N Ratio.

The problem taken in this work has 7 degrees of freedom and requires Orthogonal Array of $7 \mathrm{DOF}$, an OA with at least 7 columns is desired hence $\mathrm{L}_{8}$ is selected $\mathrm{L}_{8}$ has 7 columns and 7 DOF. Higher orthogonal arrays require more trial runs. $\mathrm{L}_{8}$ can help in experiments having interaction of parameters. Table 2 Represents L8 Orthogonal array.

\subsection{Column Assignment}

Factors were assigned to the columns as per the linear graph represented in Fig.4 Remaining factors could be assigned to any of the columns 5 or 7 in any order. Trial run calculation results are shown in table 7 . It can be seen the maximum thermal and global efficiencies are obtained in trial 4 . Maximum thermal efficiency is 8.601 and minimum is 6.746 in trial 1. Optimum combination may or may not be a part of the trial calculation. Individual average effects of different levels of parameters is shown in figure 5 .

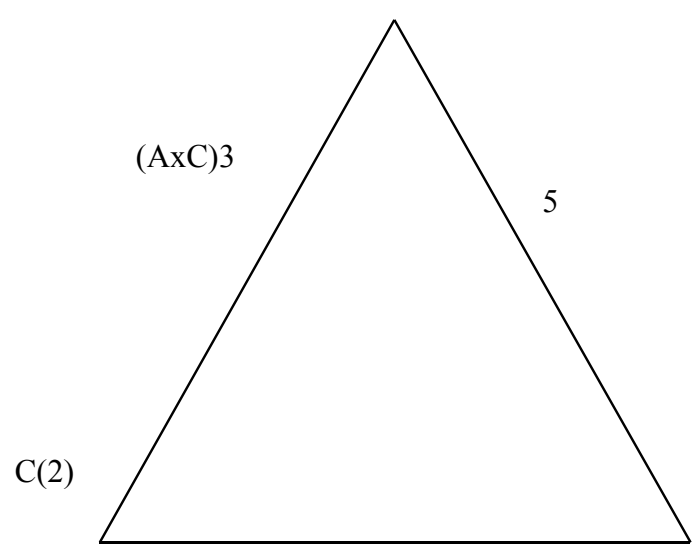

Fig.4. Linear Graph of ORC Parameters 


\subsection{Average effect of the parameters}

Average effect of level 2 of factor A is more as compared to level A, this shows that the level 2 will enhance the efficiency if used. Factor A represents turbine speed, so a higher turbine speed will result in higher efficiency of the cycle. Similarly,

Table $2: \mathrm{L}_{8}$ Orthogonal Array

\begin{tabular}{|c|c|c|c|c|c|c|c|}
\hline \multirow{2}{*}{} & \multicolumn{7}{|c|}{ FACTORS } \\
\cline { 2 - 8 } TRIAL RUN & A & C & $($ A $\times$ C $)$ & B & D & $($ B $\times$ C $)$ & E \\
\cline { 2 - 8 } & 1 & 3 & 4 & 5 & 6 & 7 \\
\hline 1 & 1 & 1 & 1 & 2 & 2 & 2 & 2 \\
\hline 2 & 1 & 2 & 2 & 1 & 1 & 2 & 2 \\
\hline 3 & 1 & 2 & 2 & 2 & 2 & 1 & 1 \\
\hline 4 & 2 & 1 & 2 & 1 & 2 & 1 & 2 \\
\hline 6 & 2 & 1 & 2 & 2 & 1 & 2 & 1 \\
\hline 7 & 2 & 2 & 1 & 1 & 2 & 2 & 1 \\
\hline 8 & 2 & 2 & 1 & 2 & 1 & 1 & 2 \\
\hline
\end{tabular}

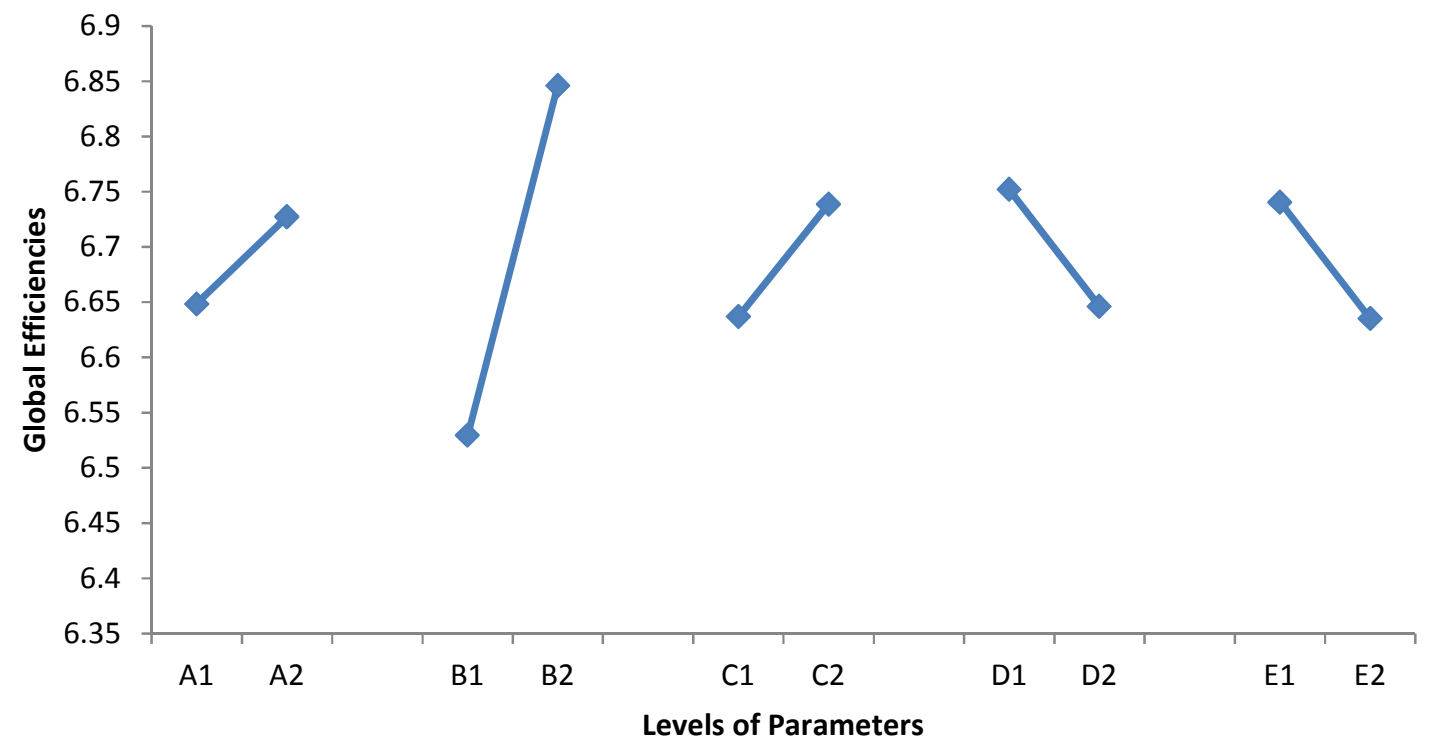

Fig. 5. Chart depicting Average effects of the parameters level 2 of factor $\mathrm{B}$, which is waste stream temperature here will be resulting in higher efficiencies. Similar effects can be found for parameters $\mathrm{C} 2, \mathrm{D} 1$ and $\mathrm{E} 1$. An optimum combination of parameters may be A2B2C2D1E1. This is optimized combination as suggested by Taguchi's approach.

Maximized efficiency is likely to be found at this combination. In the results of exhaustive calculations done on each and every combination, the efficiency at above combination shows a value of $7.202 \%$. Taguchi Calculates the optimized efficiency as

$S_{m}=\bar{Y}+\left(\overline{A_{2}}-\bar{Y}\right)+\left(\bar{B}_{2}-\bar{Y}\right)+\left(\bar{C}_{2}-\bar{Y}\right)+\left(\bar{D}_{1}-\bar{Y}\right)+$ $\left(\bar{E}_{1}-\bar{Y}\right)$

Efficiency obtained by the above relation is $7.3019 \%$. A comparison of the above shows a very less difference between the two.

\subsection{Interaction effects:}

Figure 6 shows the plot between the interaction effects of parameters $\mathrm{A}$ and $\mathrm{C}$. Two crossing lines represent that the interaction is present between two factors. Factor A and C have interactions and they effect each other. A similar curve is obtained between factor B and C as shown in figure 7 .

\section{Analysis of Variance}

The purpose of analysis of variance (ANOVA)) is to investigate which design parameter significantly affects the quality characteristic [23]. Results of ANOVA are plotted in figure 8. Waste heat temperature and the mass flow rate of waste stream affects the efficiency more than any other factor.
Levels of Parameters 
Percent effect of the interaction between the parameter A and $\mathrm{C}$ is $10 \%$ on the efficiency followed by turbine speed and condensate temperature. It is evident that waste stream temperature and its mass flow rate are two most important parameters for efficiency of ORC. Interaction effect of B and $\mathrm{C}$ has a leat effect on the efficiency.

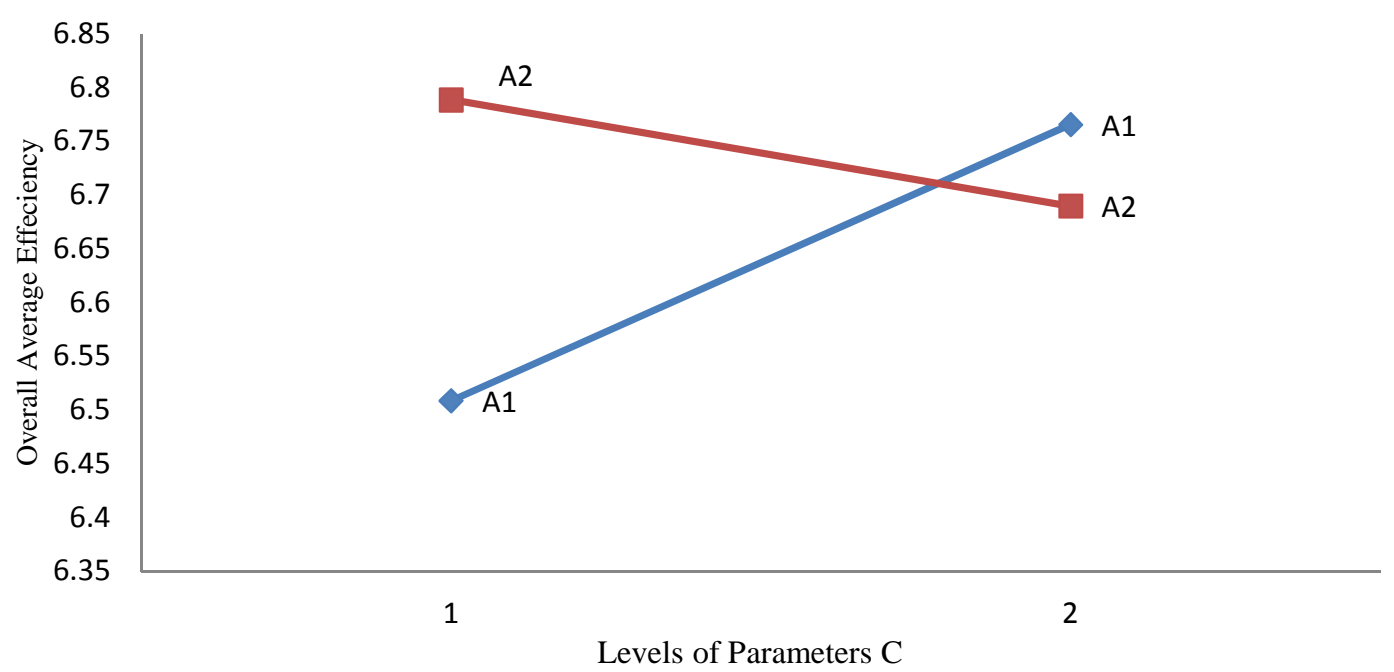

Fig. 6. Interaction of Parameter A and C

\section{RESULTS AND DISCUSSIONS}

For the Purpose of this study five parameters were selected. Results show that the efficiencies of ORC change by varying these parameters. Taguchi's approach applied to this problem tracked down these changes and maintained a quantitative account of the effect of Parameters individually as well as collectively. An optimized combination was worked out to obtain the maximum out of the available resources .

Fig. 1 represents the schematic of a typical organic Rankine cycle for refrigerant R245fa. ORC is different from water cycle in terms of operating temperatures and pressures. ORC have lower efficiencies due to narrow difference between maximum and minimum working temperatures. Parameters taken were turbine Speed, Waste Stream Temperature, Refrigerant, Mass Flow rate of waste stream, and Condensate temperature. Design of experiments was done by Taguchi's method at two levels of each parameter. L8 Orthogonal array was used. Global efficiencies of ORC were obtained and it showed that a combination of level 2 of parameters A, B and $\mathrm{C}$ and Level 1 of parameters $\mathrm{D}$ and $\mathrm{E}$ results in the optimized cycle with global efficiency of $7.2 \%$.

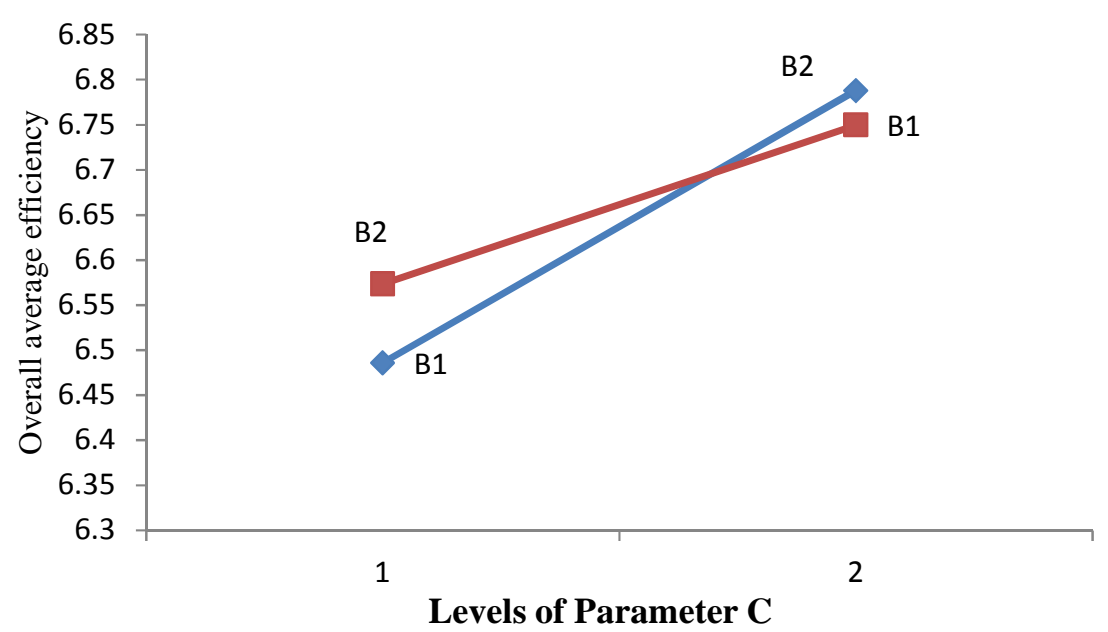

Fig.7. Inteaction effects of Parameter B and C 
Table 3 : Trial Run Calculation results

\begin{tabular}{|c|c|c|c|c|c|c|c|c|c|}
\hline \multirow[b]{3}{*}{ TRIAL RUN } & \multicolumn{9}{|c|}{ FACTORS } \\
\hline & $\mathbf{A}$ & $\mathbf{C}$ & $(\mathrm{A} \times \mathrm{C})$ & B & D & $(\mathbf{B} \times \mathbf{C})$ & $\mathbf{E}$ & \multirow{2}{*}{$\begin{array}{c}\mathbf{H}_{\text {ORC }} \\
\%\end{array}$} & \multirow{2}{*}{$\begin{array}{c}\mathbf{H}_{\text {GLOBAL }} \\
\%\end{array}$} \\
\hline & 1 & 2 & 3 & 4 & 5 & 6 & 7 & & \\
\hline 1 & 1 & 1 & 1 & 1 & 1 & 1 & 1 & 6.746 & 6.452 \\
\hline 2 & 1 & 1 & 1 & 2 & 2 & 2 & 2 & 7.228 & 6.565 \\
\hline 3 & 1 & 2 & 2 & 1 & 1 & 2 & 2 & 7.629 & 6.612 \\
\hline 4 & 1 & 2 & 2 & 2 & 2 & 1 & 1 & 8.601 & 6.965 \\
\hline 5 & 2 & 1 & 2 & 1 & 2 & 1 & 2 & 8.062 & 6.520 \\
\hline 6 & 2 & 1 & 2 & 2 & 1 & 2 & 1 & 7.011 & 6.522 \\
\hline 7 & 2 & 2 & 1 & 1 & 2 & 2 & 1 & 7.396 & 6.535 \\
\hline 8 & 2 & 2 & 1 & 2 & 1 & 1 & 2 & 7.727 & 6.844 \\
\hline
\end{tabular}

Analysis of interaction effect of parameters A and C ( Fig.7) revealed that there was a strong interaction in them, whereas parameters B and C (Fig.8) represented a weak interaction. ANOVA results gave the percentage effect of each parameter on cycle performance. Waste stream temperature and its mass flow rate have larger effect on cycle performance as compared to other parameters. Fig. 8 represents the results of ANOVA.

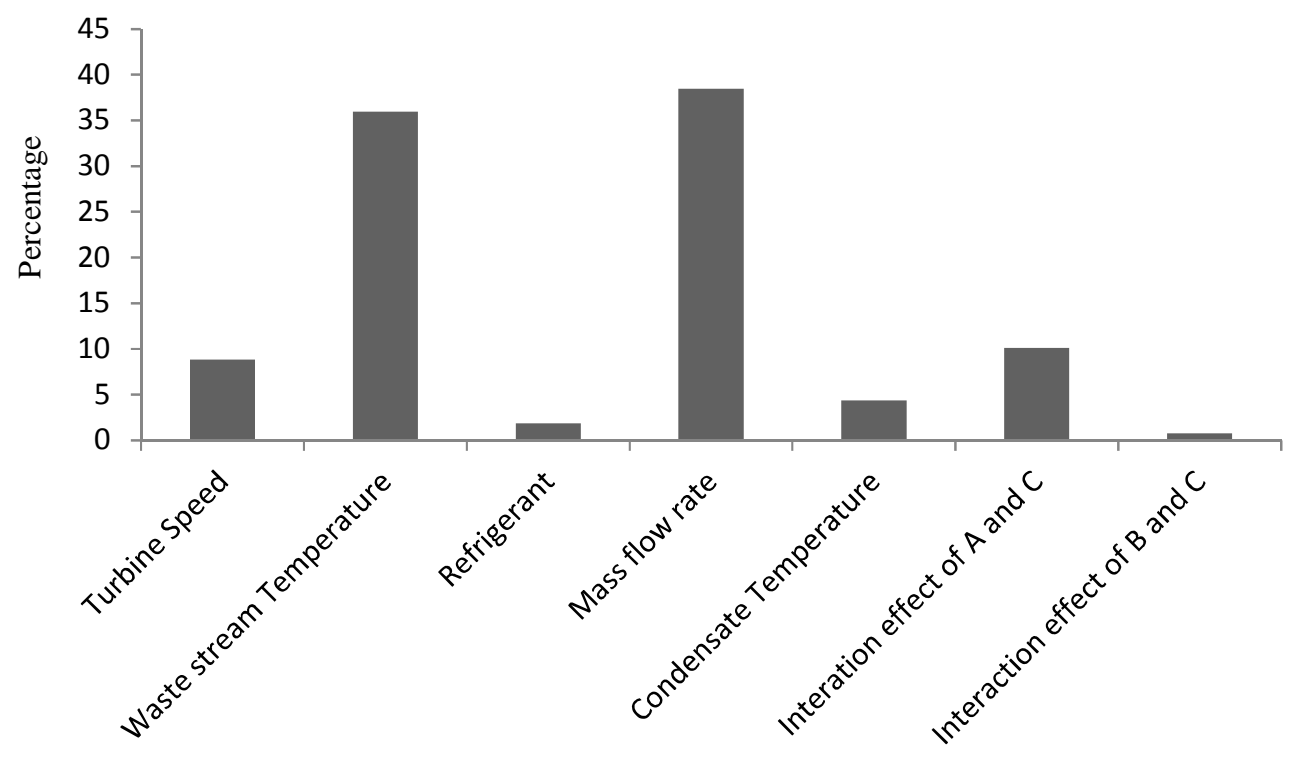

Parameters

Fig.8. Percentage effect of individual parameters 
Table 4: ANOVA table for the effect of parameters

\begin{tabular}{|c|c|c|c|c|c|c|}
\hline S.N & NAME OF PARAMETER & $\begin{array}{l}\text { COLUMN/ } \\
\text { FACTORS }\end{array}$ & DOF & $\begin{array}{l}\text { SUM OF } \\
\text { SQUARES }\end{array}$ & VARIANCE & $\begin{array}{c}\% \\
\text { EFFECT }\end{array}$ \\
\hline 1 & TURBINE SPEED & A & 1 & 0.0532 & 0.0532 & 8.857 \\
\hline 2 & $\begin{array}{l}\text { WASTE STREAM } \\
\text { TEMPERATURE }\end{array}$ & B & 1 & 0.2175 & 0.2175 & 36 \\
\hline 3 & REFRIGERANT & $\mathrm{C}$ & 1 & 0.0113 & 0.0113 & 1.881 \\
\hline 4 & MASS FLOW RATE & $\mathrm{D}$ & 1 & 0.2312 & 1.2312 & 38.49 \\
\hline 5 & $\begin{array}{l}\text { CONDENSATE } \\
\text { TEMPERATURE }\end{array}$ & $\mathrm{E}$ & 1 & 0.0261 & 0.0261 & 4.343 \\
\hline 6 & $\begin{array}{c}\text { INTERATION EFFECT OF } \\
\text { A AND C }\end{array}$ & $\mathrm{A} \times \mathrm{C}$ & 1 & 0.0609 & 0.0609 & 10.13 \\
\hline 7 & $\begin{array}{c}\text { INTERACTION EFFECT } \\
\text { OF B AND C }\end{array}$ & $\mathrm{B} \times \mathrm{C}$ & 1 & 0.0046 & 0.0046 & 0.765 \\
\hline
\end{tabular}

\section{CONCLUSIONS}

This paper has discussed an application of the Taguchi's method for optimizing the operating parameters of ORC. Limited number of researchers have tried Taguchi's approach on other than quality and manufacturing problems. Taguchi's approach applied on waste heat recovery has helped in providing a systematic and efficient methodology for optimizing the ORC cycle within available resources. Experiments were designed by using L8 Orthogonal Array for different conditions of operating parameters. Following points are drawn as conclusion of the work.

1. Taguchi's method which is commonly used in manufacturing for quality improvement, when applied to problems similar to present work can help in optimizing the system performance and thus ensuring better utilization of resources.

2. Individual as well as combined effect of parameters was assessed by using ANOVA which showed that all parameters have significant effect on cycle performance.

3. The approach is used in assigning and identifying the importance of each parameter.

4. This approach saved time and effort as the number of experiments to be performed for optimizing the cycle is very much reduced.

5. Similar types of optimization can also be attempted with large number of variables and with more levels of each variable.

\section{References:}

[1] Al-Rabgi, O.M. Beirutty, M. Akyurt, M. Najjar, Y. Alp T., Recovery and utilization of waste heat. Heat Recovery syst. CHP. 1993: 3(5): 463-470.

[2] Agustin M. Delgado-Torres, Loudes GarciaRodriguez. Analysis and optimization of low temperature solar organic Rankine cycle (ORC). Energy Conversion and Management 51(2010) 28462856.

[3] Florian Heberie, Dieter Brugeman. Exergy based fluid selection for a geothermal Organic Rankine Cycle for combined heat and power generation. Applied thermal Engineering 30( 2010) 1326-1332.

[4] Sylvain Quolin, Richard Aumann Grill, Andreas Schuster, Vincent Lemort, Hartmut Spliethoff. Dynamic Modelling and optimal control strategy of waste heat recovery Organic Rankine Cycles. Applied energy 2011; 2183-2190.

[5] Tchanche Fankam Bertrand, George Papadakis, Gregory Lambrinos, Antonios Frangoudakis. Fluid selection for a low-temperature solar organic Rankine cycle. Applied Thermal Engineering. 29, 11-12 (2009) 2468.

[6] P. J. Mago, L. M. Chamra, C Somayaji. Performance analysis of different working fluids for use in organic Rankine cycles. Journal of Power and Energy 221( 2007) 255-264. 
[7] Tianyou Wang, Yajun Zhang, Zhijun Peng, Gequn Shu. A review of researches on thermal exhaust heat recovery with rankine cycle. Renewable and sustainable Energy Reviews. 2011; 2862-2871.

[8] Adrienne B. Little, Srinivas Garimella. Comparative assessment of alternative cycles for waste heat recovery and upgrade. Energy 2011; 4492-4504.

[9] J.A Ghani, I.A. Chaudhary, H.H Hasan. Application of Taguchi method in optimization of end milling parameters. Journal of Materials Processing Technology 145 (2004) 84-92.

[10] W.H. Yang, Y.S Tarng, Design optimization of cutting parameters for turning operations based on Taguchi method, Journal of Materials Processing Technology 84(1998) 122-129.

[11] Sylvain Quoilin, Sebastien Declaye, Bertrand f. Tchanche, Vincent Lemort. Thermo-economic optimization of waste heat recovery Organic Rankine Cycles. Applied thermal Engineering XXX (2011); 19.

[12] R. E.Niggeman, W.J. Greenlee, P. Lacey. Fluid selection and optimization of an Organic Rankine cycle waste heat power conversion system, ASME 78WA, $6^{\text {th }}$ January 1978.

[13] Bertrand F. Tchanche, Gr. Lambrinos, Frangoudakis, G. Papadakis. Low-grade heat conversion into power using organic Rankine cycles - A reviewof various applications. Reneable and Sustainable Energy Reviews. 15(2011).

[14] Lee MJ, Tien DL, Shao CT. Thermophysical capability of ozone-safe working fluids for an Organic Rankine cycle system. Heat Recovey System CHP 1993;13(7):409-18.

[15] Salih Coskun, ali Riza Motorcu, Nurettin Yamankaradeniz, Erhan Pulat, Evaluation of control parameters' effects on system performance with Taguchi method in waste heat recovery application using mechanical heat pump. Inernational journal of refrigeration 35 (2012) 795-809.

[16] Kalyan K. Srinivasan, Pedro J. Mago, Sunder R Krishnan. Analysis of exhaust waste heat recovery from a dual fuel low temperature combustion engine using an Organic rankine Cycle. Energy 2010; 23872399.

[17] Jian Sun, Wenhua Li. Operation optimization of an organic rankine cycle (ORC) heat recovery power plant. Applied Thermal Engineering. 2011; 20322041.

[18] Hugues L. Talom, Asfaw Beyene. Heat Recovery from automotive engine. Applied thermal Engineering 2009; 439-444.

[19] G.S Pease, Taguchi's Methods: A hand-on approach, Addison-Wesley, Reading, M.A, 1993.

[20] G. Taguchi, Introduction to Quality engineering, Asian Productivity Organization, Tokyo,1990.

[21] A.M. Pinar, O. Kirmaci V. Optimization of counter flow Ranque-Hilsch vortex tube performance using Taguchi's method. International Journal of refrigeration 32, 1487-1494.
[22] E. Pulat, A.B. Etemoglu M. Can. Waste heat recovery potential inn Turkish Textile Industry: Case study for city of Bursa. Renewable Sustainable Energy Review. 13(3), 663-672.

[23] R.K. Roy. A primer on Taguchi Method. Competetive Manufacturing Series, USA 1990.

[24] Brandon J. Woodland, James E. Braun, Eckhard A. Groll, W. Travis Horton. Experimental Testing of an Organic Rankine Cycle with Scroll-type Expander. Purdue e pubs, Publications of the Ray W. Herrick Laboratories, Purdue University. 2505 (1-10).

[25] Dereje S. Ayou, Joan Carles Bruno, Alberto Coronas. New Power and Cooling Absorption Cycles. International Journal of Thermal \& Environmental Engineering. Volume 5, No. 2 (2013) 135-143.

\section{Nomenclature:}

$\dot{m} \quad:$ Mass flow rate, $\mathrm{kg} / \mathrm{s}$

W $\quad$ : Work, $\mathrm{kJ} / \mathrm{kg}$

$H \quad$ : Enthalpy, $\mathrm{kJ} / \mathrm{kg}$

$N \quad$ : Rotational speed, $\mathrm{rpm}$

$\mathrm{P} \quad$ : Pressure, $\mathrm{MPa}$

$W_{p} \quad$ : Pump power, $\mathrm{W}$

$W_{t} \quad$ : Turbine power, $\mathrm{W}$

h $\quad$ specific enthalpy $(\mathrm{kJ} / \mathrm{kg})$

i $\quad$ :irreversibility rate $(\mathrm{kW})$

q $\quad$ specific heat $(\mathrm{kJ} / \mathrm{kg})$

$\dot{Q} \quad$ :heat rate $(\mathrm{kW})$

$\mathrm{S} \quad$ :entropy $(\mathrm{kJ} / \mathrm{K})$

$\mathrm{T} \quad$ :temperature $(\mathrm{K})$

$\mathrm{T}_{\mathrm{H}} \quad$ : temperature of the high-temperature reservoir $(\mathrm{K})$

$\mathrm{T}_{\mathrm{L}} \quad$ : temperature of the low-temperature reservoir $(\mathrm{K})$

$\mathrm{W}$ :power $(\mathrm{kW})$

$\eta \quad$ : efficiency $(\%)$

Subscripts:

bp : boiling point

c :condenser

cycle : cycle

e : evaporator

$\mathrm{t}$ : turbine

p : : pump

o : ambient 